\title{
Rechtsgeschichte
}

\section{Gerd Bender}

\section{Vergleiche des Unvergleichbaren}


im Nationalsozialismus die Ungleichheit zu einem zentralen Prinzip des Privatrechts. Das »konkrete Ordnungsdenken « bezog Lehmann auf die gesamte Volksgemeinschaft und degradierte den Einzelnen zum Rechtsobjekt. Ähnliches verbirgt sich auch hinter dem Leitbild »soziales Recht « in der Ausprägung des Gemeinwohlgedankens. Nach 1933 wurde die Privatautonomie mehr und mehr zurückgedrängt und schließlich lediglich in ihrer "Zweckbindung " zur Durchsetzung der Belange der Volksgemeinschaft aufrechterhalten. Depping sieht damit das Privatrecht »überhaupt in seinem Bestand in Frage gestellt«. Mit der Erhebung der Rechtspflicht im Dienste der Gemeinschaft über Individualinteressen zielt Lehmann in die gleiche Richtung. In seinem Kampf gegen das subjektive Recht droht die Grenze zum öffentlichen Recht ganz zu verschwinden. Die Lektüre dieses Teils verdeutlicht erst das ganze Ausmaß des Wirkens von Lehmann im Dritten Reich: Zwar vollzog er den Abschied vom BGB formal nicht mit. Materiell verabschiedete er jedoch das Gebiet des Privatrechts an sich, indem er ihm durch Betonung der Volksgemeinschaft, auferlegte "Pflichtrechte" sowie die Annahme von Überund Unterordnungsverhältnissen die Grundlagen entzog.

Deppings Untersuchung fokussiert jedoch den Nationalsozialismus nicht übermäßig. Die Veränderungen vor I 933 und nach I 945 werden ebenso akkurat verfolgt. Zudem zieht er an entscheidenden Stellen zum Vergleich Aussagen von Zeitgenossen hinzu und lässt Lehmann nicht isoliert stehen. Nach getaner Arbeit kann der Autor nun große Schlüsse ziehen und die versprochenen dominanten Strömungen sichtbar machen: »In jedem Fall scheinen die Flexibilisierung der Privatrechtsmethode und das Eindringen sozialer Gedanken säkulare Tendenzen zu sein, mit denen Veränderungen des Privatrechts im 20. Jahrhundert umschrieben werden können. « Gute Menschen kommen in den Himmel, gute Juristen kommen überall hin.

Christine Franzius

\section{Vergleiche des Unvergleichbaren*}

Wenige Wochen nach dem vergleichsbedingten Fehltritt der Bundesjustizministerin hat sich der Hessische Ministerpräsident, ein mutmaßlicher >kommender Mann der deutschen Politik, nicht enthalten und erneut historische Parallelen ins politische Spiel gebracht. In diesem Falle ging es nicht um Bush und Hitler, sondern um laufende Vermögenssteuerdebatten und ein geschmacklos-primitives Outing reicher Leute einerseits und andererseits um die Stigmatisierung jüdischer Menschen durch den sogenannten Ju- denstern. Erneut wogte die Empörung der intellektuell-medialen Teilöffentlichkeit angesichts des >Unvergleichbaren hoch - eine Dublette an der Grenze zur Groteske, mit dem für unseren Kontext freilich nicht so wichtigen Unterschied, dass Däubler-Gmelin ging und Koch - wie auch anders - blieb. Wieder einmal, wahrlich nicht zum ersten Male, zeigte sich, wie leicht Vergleiche uns entflammen, wie dünn die Haut doch ist, wenn der Blick auf diktatorische Vergangenheiten fällt, die das Europa des 20. Jahrhunderts so
* Detlef Schmiechen-AckerMANN, Diktaturen im Vergleich (Kontroversen um die Geschichte), Darmstadt: Wissenschaftliche Buchgesellschaft 2002, VIII, I 74 S., ISBN 3-534-I4730-8;

\author{
Demokratie und Diktatur in Eu- \\ ropa. Geschichte und Wechsel der \\ politischen Systeme im 20. Jahr- \\ hundert, hg. von HeINer TIMmer- \\ MANN und WOLF D. GRUNER, \\ Berlin: Duncker \& Humblot 200I, \\ 644 S., ISBN 3-428-IO4 I 5-3
}


überreich zurückgelassen hat - sei es, dass Demokratie an Diktatur, sei es, dass Diktatur an Diktatur gemessen wird - letzteres seit dem Historikerstreit ein Dauerbrenner jeden Feuilletons.

Der letztgenannte Fall des Interdiktaturvergleichs ist Gegenstand des Buchs von Detlef Schmiechen-Ackermann. Es zeichnet sich vor allem dadurch aus, dass es auch einem breiteren Publikum Zugang verschaffen kann zu der durch allzu spezialistische Grabenkämpfe einigermaßen verunstalteten komparatistischen Diktaturforschung. Es vermittelt auf engem Raum und in prägnantem Duktus Basiswissen über die besondere Kunst des Vergleichens, und dies auf einem Feld, das wie kaum ein anderes von verbreiteter Skepsis gekennzeichnet ist. Die von Arnd Bauerkämper, Peter Steinbach und Edgar Wolfrum herausgegebene Reihe "Kontroversen um die Geschichte" hat nicht zuletzt den Historikernachwuchs zum Adressaten, dem Orientierung quer durch die weit aufgefächerte Disziplin hindurch geboten werden soll. Sie will Extrakte zu einzelnen Arbeitsbereichen der Geschichtswissenschaft zur Verfügung stellen, eine Zielsetzung, der Schmiechen-Ackermann gerecht wird.

Der methodisch orientierte Auftakt liest sich als - manchmal allzu oberseminaristisch vorgetragene - Verteidigung des Diktaturvergleichs. Wer es noch nicht wusste, wird eindringlich darüber aufgeklärt, dass jedes Vergleichen Mindestähnlichkeiten voraussetzt, nicht aber Identitätsbehauptungen, und wer es wusste, wird wieder einmal-zum wievielten Male eigentlich noch - an diesen im Grunde trivialen Sachverhalt erinnert, dessen deformierte Perzeption die Kontroversen um den Diktaturvergleich so entscheidend mitgestaltet hat.

Wie im Design der Reihe vorgeschrieben, steht eine ausführlichere Präsentation ausge- wählter Themenbereiche im Vordergrund des Buchs. Die thematische Gruppierung, die Schmiechen-Ackermann vorgenommen hat (22-I42), ist vielleicht nicht alternativenlos, macht aber, alles in allem einen vernünftigen Eindruck und charakterisiert den Stand der Forschung zutreffend. Nichts Wesentliches bleibt unberücksichtigt, die Einbeziehung der internationalen Forschung ist geglückt und ist hier mehr als mühevoll verbrämter Perspektiven-Nationalismus. Die Schwerpunkte sind » Totalitarismus<, 'politische Religionen - drei konkurrierende Deutungsmuster «, »Diktatoren und Herrschaftsapparate «, »Totalitärer Herrschaftsanspruch und Grenzen der Diktatur ", »Terror und Verfolgung «, »Opposition und Widerstand « sowie » Der integrale Vergleich von Diktaturen «. Hierbei handelt es sich - »integral « hin oder her - um Fragestellungen, die über Einzelstrukturen hinaus die Gesellschaft der Diktaturen zum Thema nehmen (56-86). »Faschistische Herrschaft: Mussolinis Italien und das >Dritte Reich`", »Der europäische Kommunismus als politische Bewegung und Herrschaftssystem «, "Nationalsozialismus und Stalinismus - die totalitären Extremformen der smodernen Diktatur « und schließlich, als steter Quell westöstlicher Workshopfreude, »NS-Regime und SED-Herrschaft: ein wichtiger Sonderfall des Diktaturenvergleichs «: es fehlt nichts, was der europäischen Zeitgeschichte gut und teuer ist, wenn sie, indem sie vergleicht, hinter die Fassaden der nationalstaatlichen Selbstinszenierungen zu schauen sucht.

Leistet Schmiechen-Ackermanns kleine Monographie, deren bibliographischen Teil (I 53I68) gerade der nur flüchtige Interessent mit großem Gewinn benutzen kann, viel Systematisches zur Situation der Forschung, wird man zumindest in diesem Punkt von »Demokratie 
und Diktatur in Europa « enttäuscht. Wie der Mitherausgeber Timmermann mitteilt, handelt es sich um einen Sammelband, der aus einer Veranstaltungsreihe des Sozialwissenschaftlichen Forschungsinstituts der Europäischen Akademie Otzenhausen »in der zweiten Hälfte der 9oer Jahre « hervorgegangen ist. Das Spektrum dessen, was das Buch über nicht weniger als 644 Buchseiten hinweg zu bieten hat, ist weit. Neben mancherlei Vermischtem zur »Demokratie" kommen auch die diversesten Aspekte der »Diktatur « zur Sprache. So reihen sich etwa ein Beitrag Karl-Egon Lönnes »Zur Demokratiekritik Gaetano Moscas « und Sybille Gerstengarbes Darstellung zur Geschichte der Leopoldina in der NS-Zeit und in der SBZ/DDR munter aneinander. Natürlich ist das allermeiste, was man bei Timmermann/Gruner lesen kann, seriös gemacht und irgendwie von Interesse - die Abhandlungen von Roland Höhne (Das Regime von Vichy. Ein europäischer Sonderfall autoritärer Herrschaft?, 473-534) und Antonio Peter (Spanien unter Franco, 445-472) zumal, werden hier doch wesentliche und alles andere als ausgeforschte Fälle bilanziert.

Insgesamt aber kann auch manch guter Aufsatz am disparaten Eindruck, den der Band hinterlässt, nichts Entscheidendes ändern. Vielmehr sieht sich das verbreitete Unbehagen an der Sammelbandkultur wieder einmal nachdrücklich bestätigt. Das Konvolut der 3 I Aufsätze ist nahe$\mathrm{zu}$ ordnungslos. Heiner Timmermann hat eine
Einführung von einer Buchseite beigesteuert, dann finden sich noch die drei Rubriken » Theorien und Begriffe", "Intertemporale und internationaleVergleiche « sowie - in unerreichbarer Prägnanz und begrifflicher Größe über fast 400 Seiten ausgreifend - »Entwicklungen in Staaten «. Das war's und das ist zu wenig, um dem Rauschen der Beiträge Struktur zu geben.

Im unkoordinierten Wechselspiel der Themen und Perspektiven wirkt Ernst Noltes Text (»Gulag « und »Auschwitz«: Unvergleichbarkeit - Parallele - kausaler Nexus?, I63-I86) wie ein Fels in der Brandung. Nicht, dass der Vater aller Schlachten, die um den Diktaturvergleich geführt werden, wirklich Neues böte. Vielmehr werden die sattsam bekannten Positionen umgewälzt und wortgewaltig verteidigt. Aber der methodologisch geladene Beitrag Noltes lässt gerade in seinen Untiefen greifbar werden, was Diktaturforschung und Diktaturvergleich bedeuten: die Auseinandersetzung mit einem Grundtatbestand des modernen Europas. Nolte lesend spüren wir, »dass die Krise der liberalen Demokratie und der damit korrespondierende Aufstieg von Diktaturen in der ersten Hälfte des Jahrhunderts (sowie deren Überwindung in der zweiten Jahrhunderthälfte) in makrohistorischer Sicht die zentralen Entwicklungsprozesse in der politischen Geschichte des 20. Jahrhunderts darstellen «(Schmiechen-Ackermann 3).

Gerd Bender 